\title{
An acute kidney injury prediction nomogram based on neurosurgical intensive care unit profiles
}

\author{
Shuo An ${ }^{1,2 \#}$, Hongliang Luo ${ }^{1,2 \#}$, Jiao Wang $^{3 \#}$, Zhitao Gong $^{1,2}$, Ye Tian ${ }^{1,2}$, Xuanhui Liu ${ }^{1,2}$, Jun Ma $^{3}$, \\ Rongcai Jiang ${ }^{1,2}$
}

${ }^{1}$ Department of Neurosurgery, Tianjin Medical University General Hospital, Tianjin 300052, China; ${ }^{2}$ Tianjin Neurological Institute, Key Laboratory of Post-Neuroinjury Neuro-repair and Regeneration in Central Nervous System, Tianjin Medical University General Hospital, Ministry of Education and Tianjin City, Tianjin 300052, China; ${ }^{3}$ Department of Health Statistics, College of Public Health, Tianjin Medical University, Tianjin 300070, China

Contributions: (I) Conception and design: R Jiang, J Ma; (II) Administrative support: S An; (III) Provision of study materials or patients: None; (IV) Collection and assembly of data: H Luo, Z Gong; (V) Data analysis and interpretation: J Wang; (VI) Manuscript writing: All authors; (VII) Final approval of manuscript: All authors.

\#These authors contributed equally to this work.

Correspondence to: Rongcai Jiang. Department of Neurosurgery, Tianjin Medical University General Hospital, 154 Anshan Road, Tianjin 300052, China. Email: jiang116216@163.com; Jun Ma. Department of Health Statistics, College of Public Health, Tianjin Medical University, No. 22 Qixiangtai Road, Heping District, Tianjin 300070, China. Email: junma@tmu.edu.cn.

Background: Acute kidney injury (AKI) is a common and serious complication with high mortality within the neural-critical care unit, and can limit the treatment of osmotic diuresis and body fluid equilibrium. Given its seriousness, it is necessary to find a tool to predict the likelihood of AKI and to prevent its occurrence.

Methods: In this retrospective study, patients' clinical profiles, laboratory test results, and doctors' prescriptions were collected. Least absolute shrinkage and selection operator (LASSO) regression was used to select variables, and a logistic regression model was then applied to find independent risk factors for AKI. Based on the results of multivariate analysis, we established a nomogram to evaluate the probability of AKI, which was verified through the use of a receiver operating characteristic (ROC) curve and its calibration curves.

Results: Risk factors given by logistic regression were Glasgow Coma Scale (GCS) classification (1.593; 95\% CI: 0.995-2.549; P=0.0523), coefficient of variation (CV) of GCS (1.017; 95\% CI: 0.995-1.04; $\mathrm{P}=0.1367)$, hypertension (2.238; 95\% CI: 1.124-4.456; $\mathrm{P}=0.0219)$, coronary heart disease (2.924; $95 \% \mathrm{CI}$ : 1.2-7.126; $\mathrm{P}=0.0182)$, pneumonia within 7 days (3.032; 95\% CI: $1.511-6.085 ; \mathrm{P}=0.0018)$, heart failure within 7 days (6.589; 95\% CI: 2.235-19.42; $\mathrm{P}=0.0006)$, furosemide (1.011; 95\% CI: 1.005-1.016; $\mathrm{P}<0.0001)$, torasemide $(1.028 ; 95 \% \mathrm{CI}: 0.976-1.082 ; \mathrm{P}=0.297)$, dopamine (1; 95\% CI: $1-1.001, \mathrm{P}=0.3297)$, and norepinephrine (1.007; 95\% CI: $1-1.015 ; \mathrm{P}=0.0474)$. The area under the curve (AUC) of the prediction model was 0.8786 , and the calibration curves showed that the model had a good ability to predict AKI occurrence.

Conclusions: This study presents an AKI prediction nomogram based on LASSO, logistic regression, and clinical risk factors. The clinical use of the nomogram may allow for the timely detection of AKI occurrence and thus improve the prognosis of patients.

Keywords: Neurosurgical intensive care; acute kidney injury (AKI); risk factors; nomogram

Submitted Oct 14, 2019. Accepted for publication Dec 20, 2019.

doi: $10.21037 /$ atm.2020.01.60

View this article at: http://dx.doi.org/10.21037/atm.2020.01.60 


\section{Introduction}

The development of neurosurgery/neurological critical care medicine has an over 50-year history. The purpose of this care is to provide advanced life support and effective solutions to treat traumatic and non-traumatic acute central nervous system injury. Given the severe conditions of the patients and the specialized nature of the medical treatment in neurosurgery/neurological intensive care units (NICU), serious complications are to be expected when compared with patients in regular wards. Gruber et al. found that $81 \%$ of patients suffer from at least 1 additional organ dysfunction apart from the central nervous system (1). The commonly used medications in the NICU, including mannitol, vancomycin, and various contrast agents, are nephrotoxic, which limits the options of drug therapy when acute kidney injury (AKI) occurs. This is of particular concern as it is essential to prevent the occurrence of renal dysfunction, (also known as AKI), at as early a stage as possible. However, physicians and surgeons may be indecisive or reluctant in choosing a given medication or its dosage, and thus outcomes can be adversely affected.

$\mathrm{AKI}$, as a worldwide health issue, is considered to be a complication with high mortality and poor prognosis. Previous studies have shown the AKI incidence range dramatically from between $0.7 \%$ and $77.2 \%$ depending on the different diagnostic standards and study cohorts (2-4). According to Büttner's research, this figure was $11.6 \%$ in neurocritical care settings (5), while the mortality of AKI patients has been reported to be more than 16 per 100 person-years (6), and increasing. Based on the verification of traditional risk factors, we designed a new, retrospective study to identify new risk factors and their related effects according to different clinical profiles. Specifically, we aimed to determine the risk factors of AKI development and create a scale to evaluate the probability for patients undergoing critical neurosurgical care to suffer from AKI.

\section{Methods}

\section{Patient selection}

In the NICU of Tianjin Medical University General Hospital (which is the highest-level hospital located in the center of the municipality of Tianjin), we admit patients with life-threatening acute brain injury (ABI) with or without complications. This includes patients who suffer from traumatic brain injury, intracerebral hemorrhage, subarachnoid hemorrhage, post-operation, and other acute

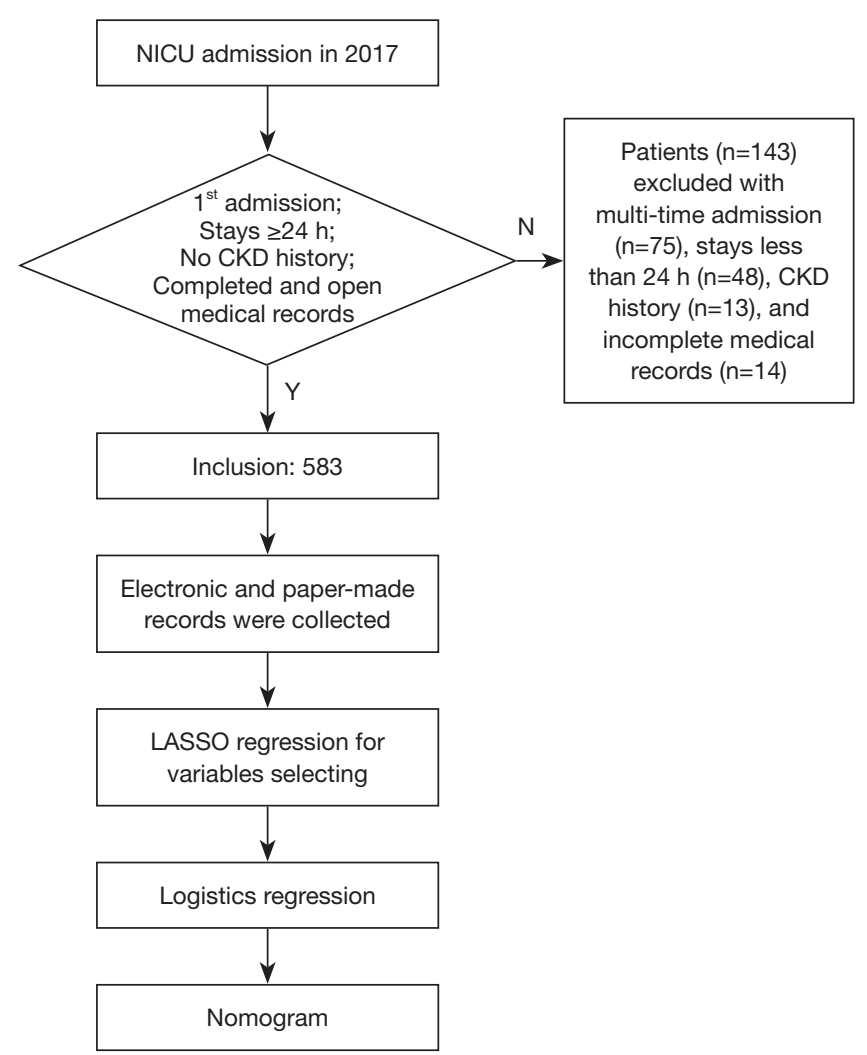

Figure 1 Study flow chart. LASSO, least absolute shrinkage and selection operator.

central nervous system injury. With the support of the consulting protocol, any non-neurosurgery issue is solved in cooperation with the other relevant department. The ABI patients who met the standard of our NICU admission and who were admitted to the NICU between January 2017 and December 2017 were included in this retrospective study. Patients who stayed in the NICU less than 24 hours, who had been hospitalized multiple times, or who had been diagnosed with chronic kidney disease (CKD), including those found with renal dysfunction within the last 3 months (as determined by their medical histories) were excluded (Figure 1). This study was approved by the ethics committees of Tianjin Medical University General Hospital.

\section{Data collection}

We retrospectively collected the patients' data, including demographics, laboratory parameters, pharmacy prescription details, and surgery and operation information. Patients were monitored during their hospital stays. We 
used the highest value of the Glasgow Coma Scale (GCS) per hour of every day to assess impaired consciousness and coma (7). For blood pressure (BP), invasive BP and noninvasive $\mathrm{BP}$ were measured and recorded by the hour. We recorded non-invasive systolic pressure and diastolic pressure separately. Intake and output volume were recorded every 24 hours. The coefficient of variation (CV) and mean were calculated for each of GCS, BP, and intake/ output value. We also collected patients' medical history, particularly information related to diabetes, coronary artery disease, and hypertension. Laboratory test results during patients' hospital stay, including serum creatine (SCr), aspartate aminotransferase (AST), alanine aminotransferase (ALT), white blood cell (WBC) count, and other values were recorded. We recorded the baselines of these tests with the first value being within 3 days of onset admission.

\section{Definition of AKI}

The outcome was the occurrence of AKI during the patients' hospital stay, according to the Kidney Disease: Improving Global Outcomes (KDIGO) guidelines (8). In this study, once a patient met the standard of stage 1 (serum creatinine, $1.5-1.9$ times baseline or $\geq 26.5 \mu \mathrm{mol} / \mathrm{L}$ increase; urine output, $<0.5 \mathrm{~mL} / \mathrm{kg} / \mathrm{h}$ for $6-12$ hours), we placed that patient into the AKI group.

\section{Statistical analysis}

Numerical variables of the normal distribution are expressed as mean $\pm \mathrm{SD}$, while those of non-normal distribution are expressed as median (quartile). The continuous data and category data between the AKI and non-AKI groups were compared by Wilcoxon rank-sum test and Chi-square test, respectively. The significance level of the above statistical analyses was set as $\alpha=0.05$, and $\mathrm{P}<0.05$ (two-tailed) was considered statistically significant. Least absolute shrinkage and selection operator (LASSO) regularization was used for variable selection, and logistic regression was used to estimate the association of independent risk factors and AKI. LASSO regression is a compression estimation that is used to deal with the collinearity between covariates. When there are several collinear predictors, LASSO selects only one and ignores the others or zeroes out some regression coefficients. In this study, the training set and the validation set were divided into a 7:3 ratio to find the optimal LASSO model. Multicollinearity was tested using the variance inflation factor (VIF) method, with a VIF $\geq 10$ indicating the presence of several multicollinearities. Finally, we built a nomogram based on the results of a logistic regression model, which was verified through the use of the HosmerLemeshow tests and the calibration curve. All statistical analyses were performed using SAS 9.4 and R software (version 3.5.1; http://www.r-project.org/) (packages: ResourceSelection, rms, ggplot2).

\section{Results}

In total, 583 patients were enrolled in this study, and 143 patients were excluded. Seventy-one patients had AKI, meaning that the incidence of AKI was $12.17 \%$. With the median age of 66 years, over six-tenths $(64.79 \%)$ of the patients were male. Compared with non-AKI patients, patients with AKI were significantly older $(\mathrm{P}=0.001)$, while their mortality and NICU stays were significantly higher and longer $(\mathrm{P}<0.0001)$. Table 1 shows a comparison of the significant characteristics of patients enrolled in the study $(\mathrm{P}<0.05)$. The proportions of main diagnoses, medical history, the occurrences of comorbidities, etc. were significantly different $(\mathrm{P}<0.05)$.

The baselines of laboratory tests and drug usage are shown in Table 2 and Table 3, respectively. AKI patients tended to have significantly higher alkaline phosphatase (ALP), blood potassium concentration $\left(\mathrm{K}^{+}\right)$, and lower blood platelet (PLT) levels $(\mathrm{P}<0.05)$. Differences in drug administration were also observed, with AKI patients having significantly more use of antibiotics, including meropenem, piperacillin sodium tazobataner sodium (PSTS), cefoperazone sodium, and sulbactam sodium (CSSS) $(\mathrm{P}<0.05)$. Compared with non-AKI patients, vasopressor agents like dopamine and noradrenaline were significantly more prescribed in AKI patients $(\mathrm{P}<0.05)$. All the other significant differences in drugs including that of fasudil, furosemide, torasemide, etc. can be seen in Table $2(\mathrm{P}<0.05)$.

To confirm the independent risk factors of AKI, we performed LASSO regularization to select variables (Figure 2) and followed this with logistics regression analysis. Table 4 shows the results of multivariate analysis. With a poorer GCS classification (1.593; 95\% CI: 0.995-2.549), higher CV of GCS (1.017; 95\% CI: 0.995-1.04), hypertension (2.238; 95\% CI: $1.124-4.456)$, coronary heart disease (CHD) (2.924; 95\% CI: 1.2-7.126), pneumonia diagnosed within 7 days (3.032; 95\% CI: 1.511-6.085), and heart failure within 7 days (6.589; 95\% CI: 2.235-19.42), along with greater use of furosemide (1.011; 95\% CI: $1.005-$ 1.016), torasemide (1.028; 95\% CI: 0.976-1.082), dopamine 
Table 1 Characteristics of patients enrolled in this study

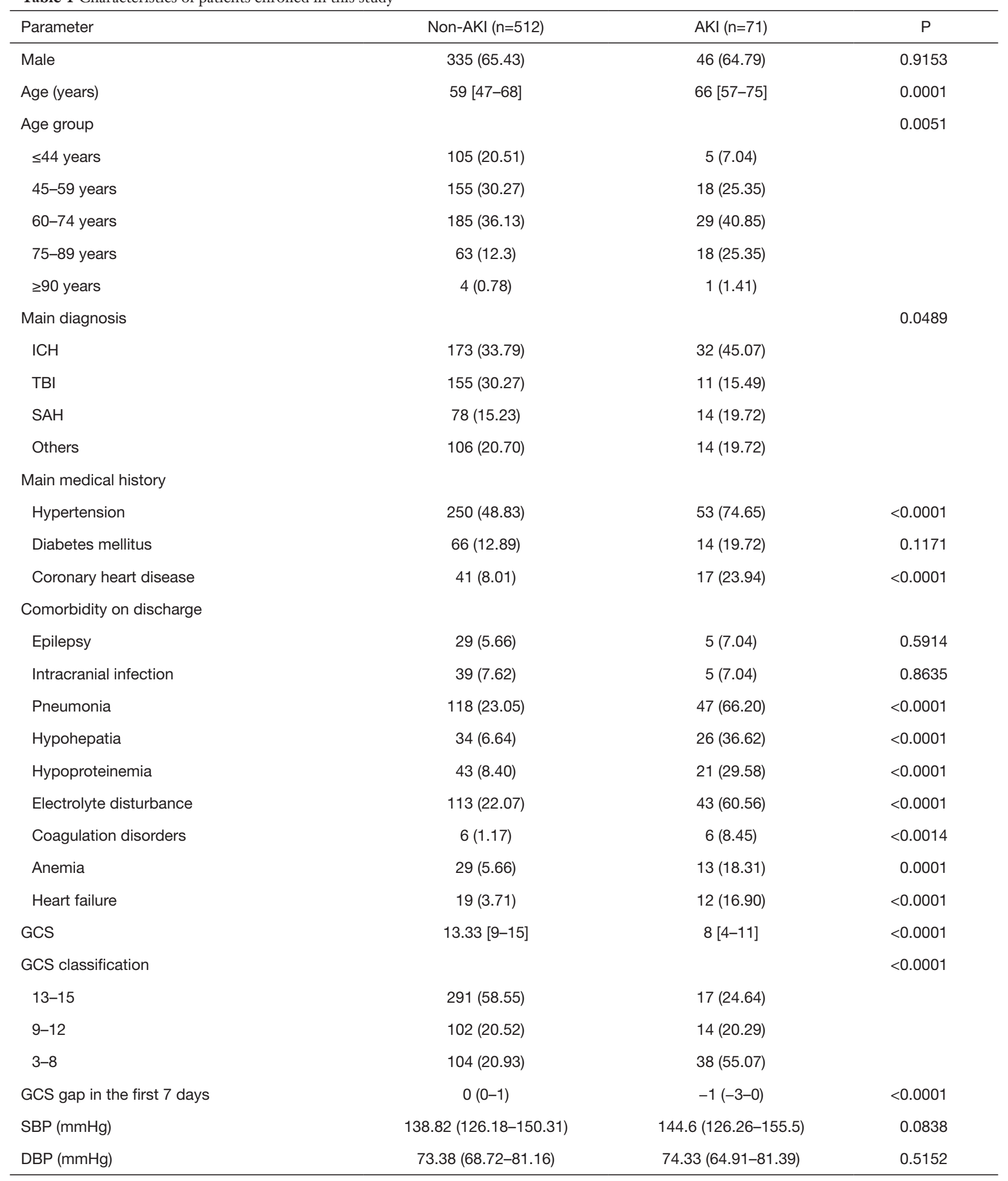

Table 1 (continued) 
Table 1 (continued)

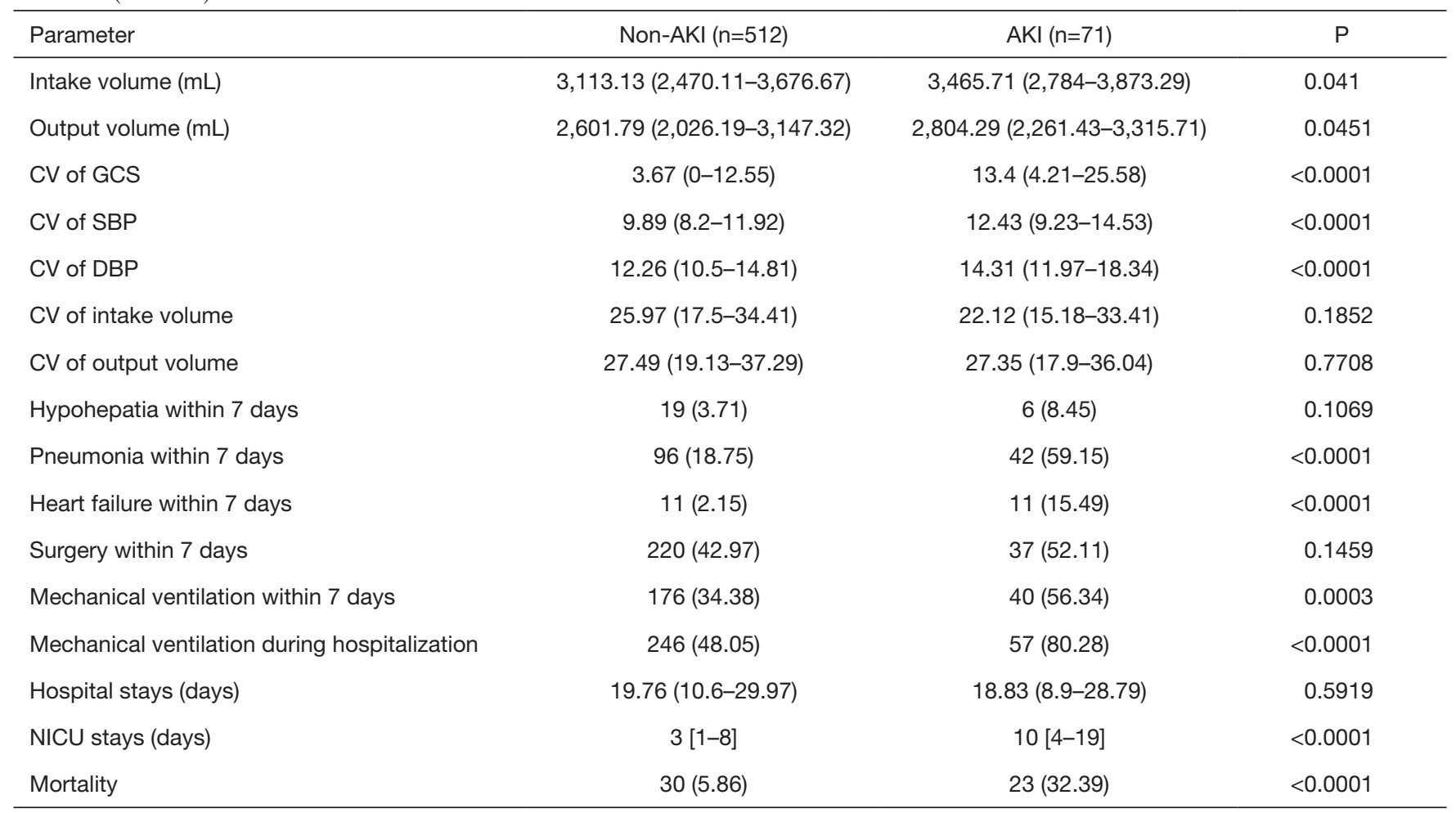

Data are shown as number (percentage) or median (quartile). AKI, acute kidney injury; $\mathrm{ICH}$, intracranial haemorrhage; TBI, traumatic brain injury; SAH, subarachnoid haemorrhage; GCS, Glasgow coma scale; SBP, systolic blood pressure; DBP, diastolic blood pressure; CV, coefficient of variation; NICU, neurosurgery intensive care unit.

(1; 95\% CI: $1-1.001)$, and norepinephrine (1.007; 95\% CI: $1-1.015)$, the patients would be more likely to suffer from AKI. However, a higher GCS (0.955; 95\% CI: 0.848-1.074) showed a reduced likelihood of AKI occurrence.

Figure 3 is the nomogram of the logistic regression model. All selected variables have their own lines in the nomogram, with each receiving a point according to the value and the scale at the top of the figure. Total points were added up, and the probability of AKI occurrence was matched. Figure 4 is the calibration curve. The apparent value is close to the bias-corrected value. The HosmerLemeshow tests proved the good predictive performance of the model $\left(\chi^{2}=4.4175\right.$ and $\mathrm{P}$ value $\left.=0.8176\right)$. According to the above picture and area under the ROC curve (AUC $=0.8786$ ) (Figure 5), the model obtained in this study has a good predictive performance for AKI.

\section{Discussion}

Currently, there is no effective practical tool for estimating the likelihood of AKI occurrence after acute brain injury, especially in the NICU. To redress this shortcoming, we conducted a study based on NICU patients' clinical profiles and effective mathematical modelling methods. This risk score system in the study is easy and convenient to apply to early stage AKI prediction in the NICU. It may help clinical physicians detect AKI and manage it in a timely manner. Based on different regressions, we found 10 risk factors of AKI occurrence in this retrospective study, including GCS, $\mathrm{CV}$, hypertension, $\mathrm{CHD}$, and pneumonia and heart failure within the first week, along with the use of furosemide, torasemide, dopamine, and noradrenaline. GCS difference was performed as a protective factor in our analysis.

In the NICU, the GCS scoring system is commonly used to evaluate the severity of a patient's condition. In our study, based on hourly GCS scoring, we calculated the mean GCS value in the first week to estimate the condition of the patients. We also calculated the coefficient of variation to observe the stability of consciousness and the value gap between the seventh day after admission and admission day. Results revealed that the patients with a low GCS mean value and a high $\mathrm{CV}$ were more likely to suffer from AKI. 
Table 2 Baselines of laboratory test values

\begin{tabular}{|c|c|c|c|c|}
\hline Parameters & Non-AKI (n=512) & $A K I(n=71)$ & Z & $\mathrm{P}$ \\
\hline ALP (U/L) & 74 [62-93] & 81 [71-98] & 2.015 & 0.0439 \\
\hline ALT (U/L) & 33 [22-44] & 33 [26-41] & 0.1472 & 0.883 \\
\hline AST (U/L) & 30 [23-42] & 30 [25-41] & 0.0004 & 0.9997 \\
\hline $\mathrm{Hb}(\mathrm{g} / \mathrm{L})$ & 135 [122-147] & 134 [122-145] & -0.1571 & 0.8751 \\
\hline WBC $\left(\times 10^{9} / \mathrm{L}\right)$ & $10.44(7.62-14.38)$ & 10.59 (7.49-14.81) & 0.1869 & 0.8518 \\
\hline $\operatorname{PLT}\left(\times 10^{9} / \mathrm{L}\right)$ & 207 (168.5-258) & 180 (147.5-226) & -3.2057 & 0.0013 \\
\hline L\% & $9.6(5.85-16.3)$ & $8.85(5.8-15.2)$ & -1.0739 & 0.2829 \\
\hline PT (s) & $11.1(10.5-11.8)$ & $10.9(10.4-12.4)$ & 0.1486 & 0.8819 \\
\hline PT-INR & $1.01(0.96-1.08)$ & $1(0.95-1.13)$ & 0.143 & 0.8863 \\
\hline APTT (s) & 28.5 (26.4-31.35) & $28.6(26.5-31.4)$ & -0.1174 & 0.9066 \\
\hline$\pi(s)$ & $19.1(17.8-20.4)$ & $19.15(18.1-20.5)$ & 0.4431 & 0.6577 \\
\hline FIB (g/L) & $3.04(2.55-3.67)$ & $3.37(2.86-3.8)$ & 1.8829 & 0.0597 \\
\hline D-Dimer (ng/mL) & $1,371[459-5,825]$ & $1,102.5[537-4,812]$ & 0.0319 & 0.9745 \\
\hline $\mathrm{Na}^{+}(\mathrm{mmol} / \mathrm{L})$ & 140 [138-142] & 140 [138-143] & 0.2625 & 0.7929 \\
\hline $\mathrm{K}^{+}(\mathrm{mmol} / \mathrm{L})$ & $3.7(3.4-4)$ & $3.6(3.2-3.9)$ & -2.2212 & 0.0263 \\
\hline
\end{tabular}

Data are shown as median (quartile). AKI, acute kidney injury; ALB, albumin; ALP, alkaline phosphatase; ALT, alanine transaminase; AST, aspartate transaminase; GGT, gamma-glutamyl transpeptidase; Hb, hemoglobin; WBC, white blood cell; PLT, platelet; L\%, percentage of lymphocytes; N\%, percentage of neutrophil granulocyte; TBIL, total bilirubin; PT, prothrombin time; INR, international normalized ratio; APTT, activated partial thromboplastin time; TT, thrombin time; FIB, fibrinogen; $\mathrm{Na}^{+}$, sodium concentration; $\mathrm{K}^{+}$, potassium concentration.

Table 3 Use of medicines

\begin{tabular}{|c|c|c|c|c|}
\hline Parameter & Non-AKI (n=512) & AKI $(n=71)$ & Z & $\mathrm{P}$ \\
\hline Diazepam (IV, mg) & $2.19 \pm 4.89$ & $1.13 \pm 3.18$ & -1.6056 & 0.1084 \\
\hline Dezocine (IV, mg) & $0.36 \pm 3.58$ & $0.42 \pm 2.64$ & 0.2552 & 0.7986 \\
\hline Remifentanil (IV, mg) & $0.04 \pm 0.35$ & $0.06 \pm 0.29$ & 1.5297 & 0.1261 \\
\hline Dexmedetomidine (IV, mL) & $2.46 \pm 6.63$ & $3.46 \pm 7.1$ & 1.5477 & 0.1217 \\
\hline \multicolumn{5}{|l|}{ Antibiotics } \\
\hline Biapenem (IV, g) & $0.06 \pm 0.57$ & $0 \pm 0$ & -0.8332 & 0.4047 \\
\hline
\end{tabular}

Table 3 (continued) 
Table 3 (continued)

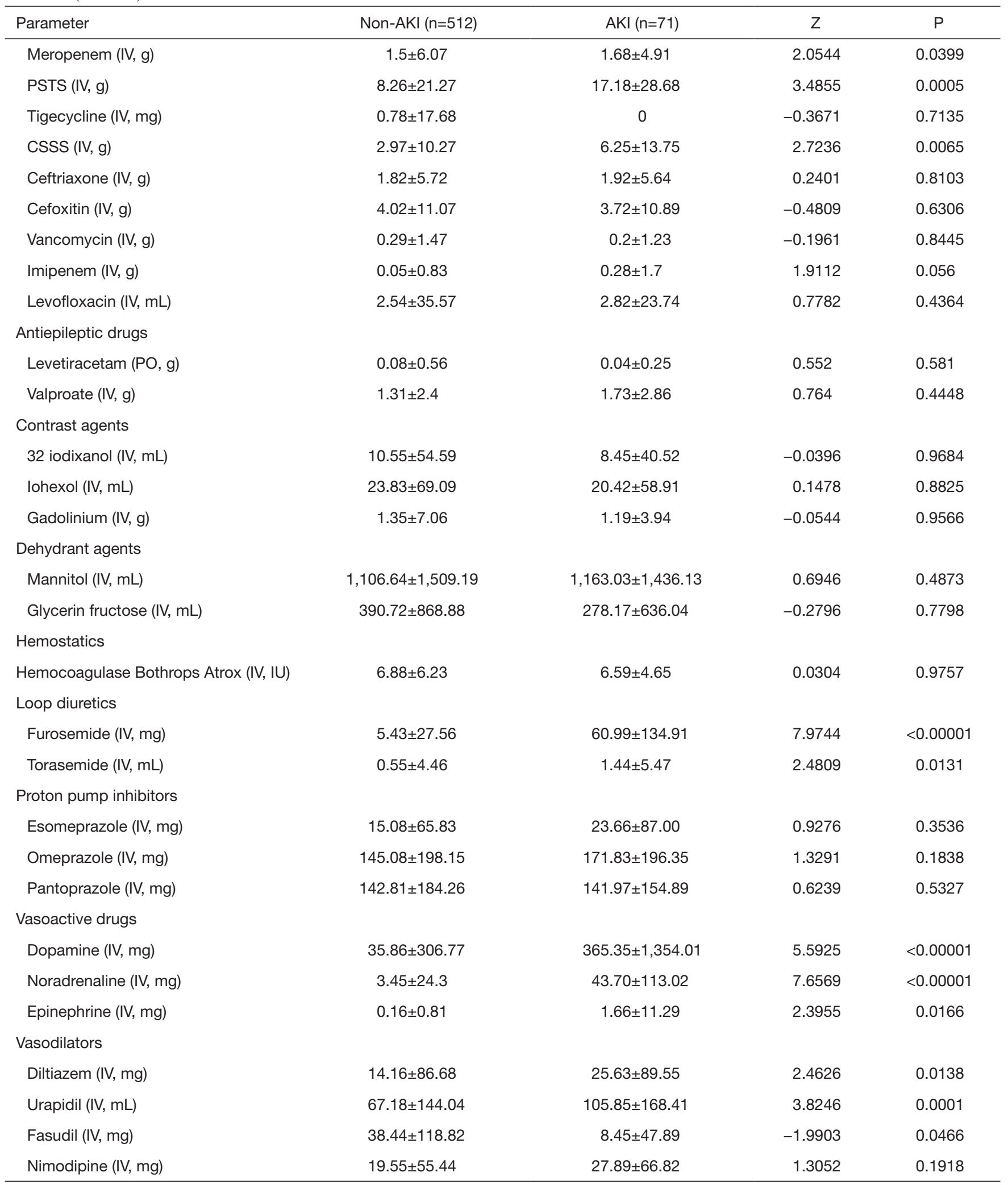

Data are shown as mean \pm SD. AKI, acute kidney injury; PSTS, piperacillin sodium tazobactam sodium; CSSS, cefoperazone sodium sulbactam sodium. 


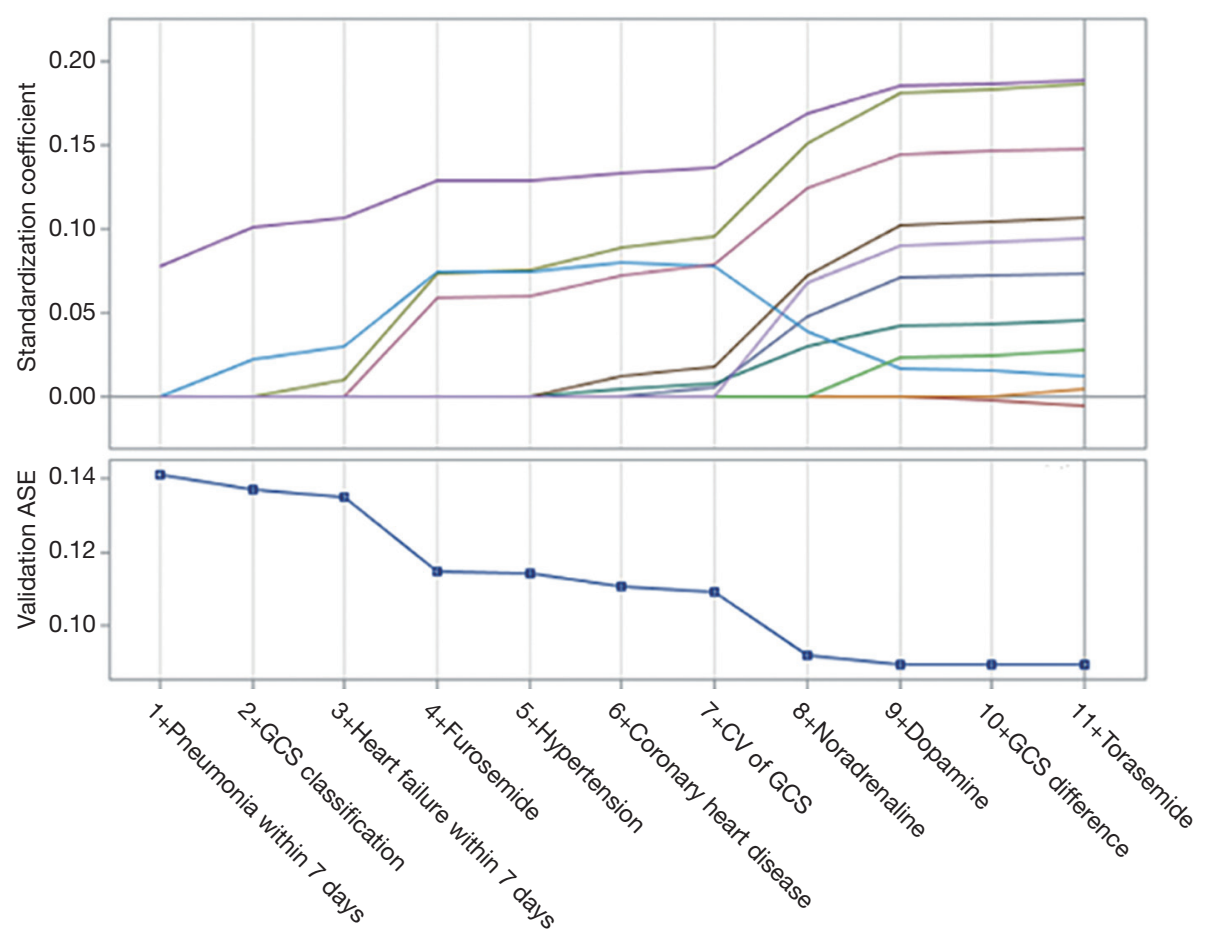

Figure 2 Least absolute shrinkage and selection operator (LASSO) coefficient profiles of variables. The figure illustrates the selection process of LASSO. The abscissa stands for the step-by-step selection of variables. Eleven variables were admitted to the final model based on the minimum average square error (ASE).

In contrast, the probability of AKI decreased the higher the GCS value gap was.

Our study highlights medical histories of coronary heart disease and hypertension as risk factors, which corresponds to the significant difference in the age of AKI patients $(\mathrm{P}=0.001)$. CHD has been reported to be associated with acute or chronic kidney injury (9). Furthermore, the connection between hypertension and weaker renal function has been known since the first description of untreated hypertensive vascular disease (10).

Infection, especially sepsis, is the most important risk factor for renal function. In the NICU, infection is mainly caused by pneumonia (lung infection) which is related to a disturbance of consciousness, immune dysfunction, or a weaker bucking response of the patients. Mechanical ventilation $(\mathrm{MV})$ is the most commonly used medical treatment in the NICU, especially for serious cases that require externally assisted breathing. Our analysis indicates that MV is more often used in AKI patients who also have a high incidence of pneumonia $(\mathrm{P}<0.01)$.

Although use of antibiotics medicine was not found to be significant in our multivariate analysis result based on 7-day treatment data, the univariate analysis did show some significant differences between AKI patients and non-AKI patients for piperacillin sodium tazobataner sodium (PSTS), cefoperazone sodium and sulbactam sodium (CSSS), and meropenem $(\mathrm{P}<0.01)$. There are several reports which state that PSTS can induce renal dysfunction $(11,12)$.

Neither the univariate analysis nor the final model of our study found a significant difference with regards to the effect of vancomycin, which was thought to be nephrotoxic, on AKI patients. This might be because there were only a few patients who were treated with vancomycin to prevent infection in the first 7 days after admission, and physicians preferred to use other less nephrotoxic drugs when there was a high risk for AKI.

Mannitol is the most commonly used dehydration drug in the NICU, and it is nephrotoxic. Interestingly, mannitol was not admitted into our final model. However, diuretics were analyzed as a risk factor. Diuretics are used by physicians and surgeons as a replacement treatment for intravenous injection of mannitol, in cases when AKI occurs or when the baseline creatinine exceeds the upper limit value. However, it has been proven that the use of diuretics may increase the 
Table 4 The multivariable analysis of AKI

\begin{tabular}{|c|c|c|c|c|c|}
\hline Parameters & \multicolumn{2}{|c|}{ LASSO regression } & \multicolumn{3}{|c|}{ Logistic regression } \\
\hline GCS classification & 0.002336 & 0.012426 & 0.4655 & $1.593(0.995-2.549)$ & 0.0523 \\
\hline GCS difference & -0.000698 & -0.005289 & -0.0466 & $0.955(0.848-1.074)$ & 0.4382 \\
\hline CV of GCS & 0.001484 & 0.073869 & 0.0168 & $1.017(0.995-1.04)$ & 0.1367 \\
\hline Coronary heart disease & 0.129875 & 0.107328 & 1.0730 & $2.924(1.2-7.126)$ & 0.0182 \\
\hline Pneumonia within 7 days & 0.127576 & 0.189016 & 1.1094 & $3.032(1.511-6.085)$ & 0.0018 \\
\hline Heart failure with 7 days & 0.344287 & 0.18626 & 1.8854 & $6.589(2.235-19.42)$ & 0.0006 \\
\hline Furosemide & 0.001251 & 0.148214 & 0.0106 & $1.011(1.005-1.016)$ & $<0.0001$ \\
\hline Norepinephrine & 0.000976 & 0.09422 & 0.0073 & $1.007(1-1.015)$ & 0.0474 \\
\hline
\end{tabular}

AKI, acute kidney injury; LASSO, least absolute shrinkage and selection operator; GCS, Glasgow coma scale. CV, coefficient of variation.

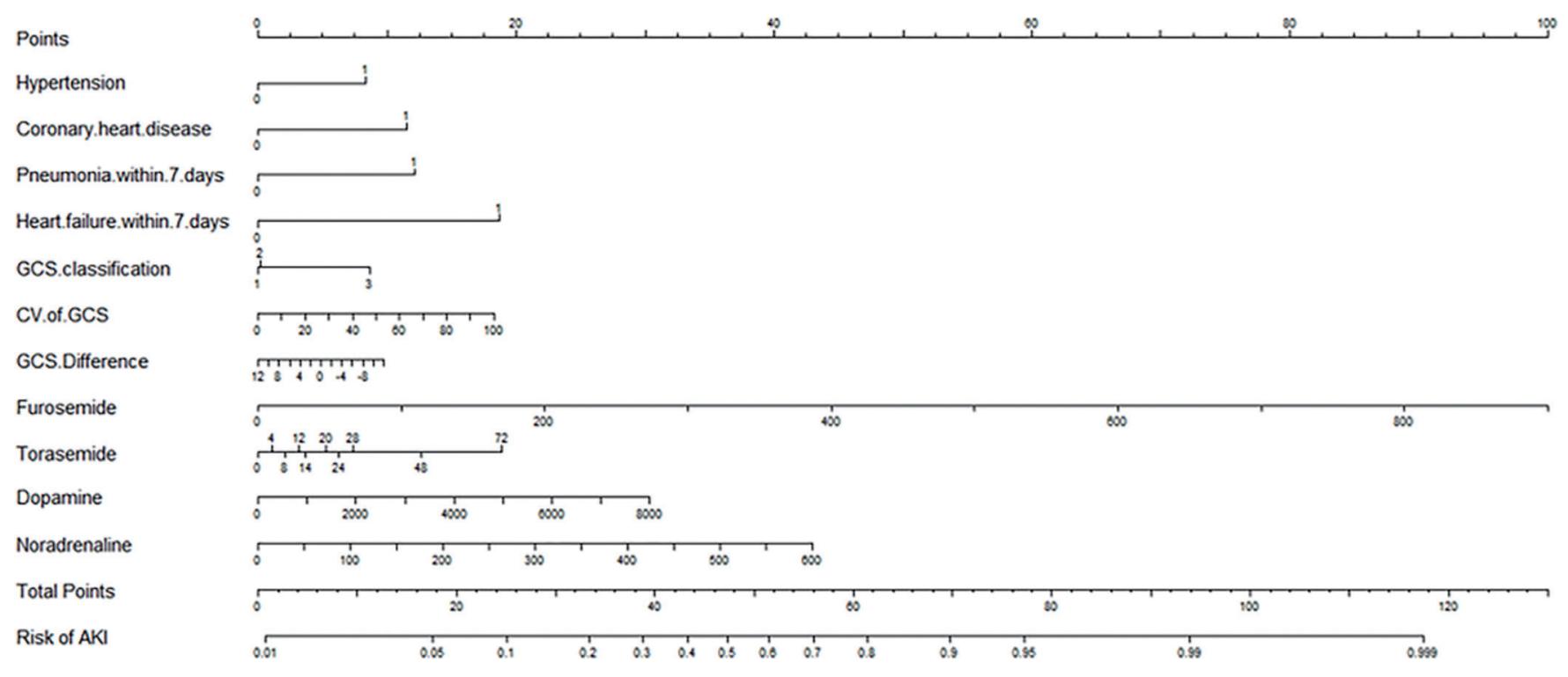

Figure 3 The nomogram developed by logistic regression. Each selected variable is represented by a line in the figure. According to the value, each variable receives a point. Total points are added for each variable and matched with the probability of AKI occurrence. AKI, acute kidney injury.

risk of AKI, especially loop diuretics (13-15).

Norepinephrine and dopamine, which are used to treat hypotension, were key risk factors in our model. Norepinephrine was thought to decrease the blood flow of visceral organs, like the kidney, when vascular beds constrict under normal conditions. Yet, the latest research has indicated that, under fluid-resuscitated endotoxemic conditions, renal oxygen delivery, gross filtration rate (GFR), and renal oxygen supply can be improved by using norepinephrine to restore blood pressure in cases of vasodilatory shock and AKI (16). Low dose dopamine (LDD) has been used for its renal blood flow and urine 


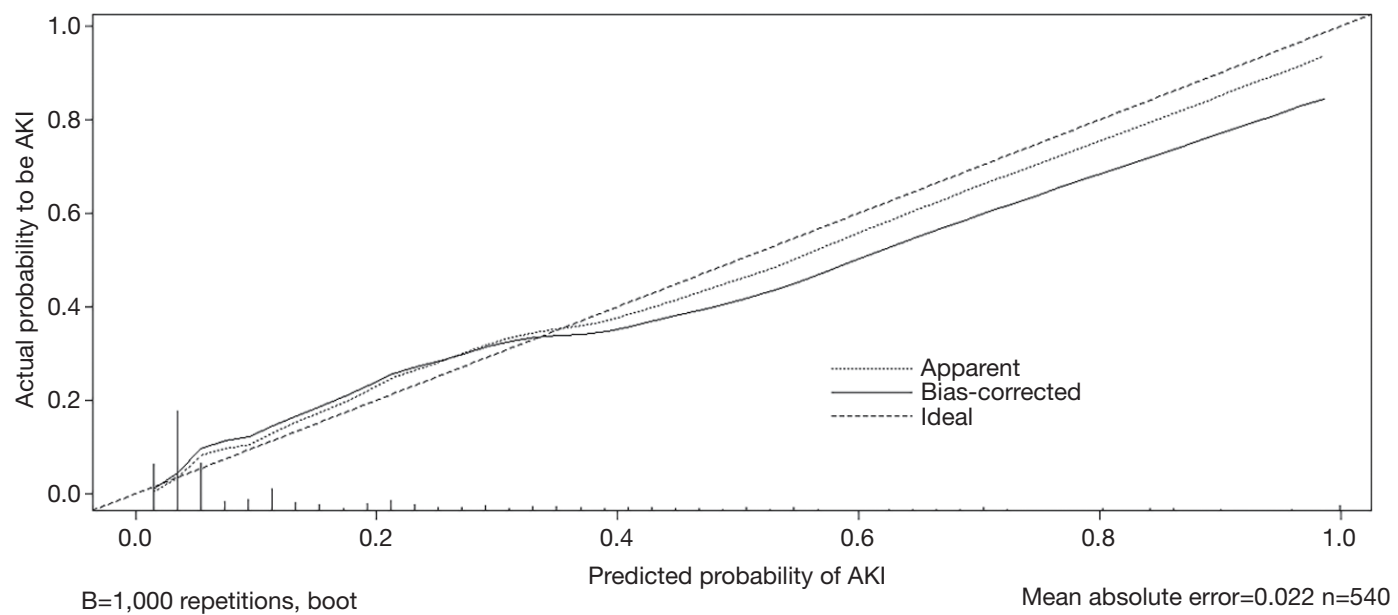

Figure 4 Calibration curves of the nomogram. The lines in the figure represent the apparent value, the bias corrected value, and the ideal value. The apparent and the bias-corrected values are close to each other, which means the nomogram has a good predictive performance. The good performance of the model can also be confirmed by the Hosmer-Lemeshow test $\left(\chi^{2}=4.4175\right.$ and $\mathrm{P}$ value $\left.=0.8176\right)$.

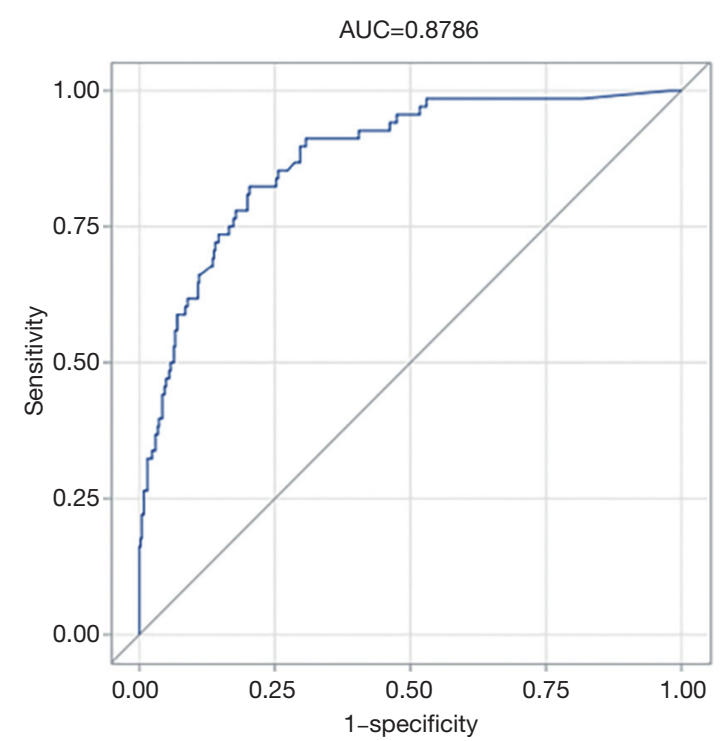

Figure 5 The ROC curve. The area under the receiver operating characteristic (AUC) is 0.8786 .

output improvement benefits, but lately, its use has become controversial. Some researchers have concluded that LDD plays no role in improving renal function or preventing acute renal insufficiency $(17,18)$. KIDGO has no recommendations concerning the use of dopamine in its new guidelines (8). Based on this knowledge, we considered that, although these are risk factors, the probable explanation relates principally to hypotension, and thus norepinephrine can be first chosen over dopamine to maintain blood pressure.

In addition, these findings, some limitations to our study should be addressed. Firstly, this is an observational study that cannot directly draw causal conclusions. Secondly, considering the mean of AKI occurrence time was around 7 days after admission, we only collect the data for a week after the onset of admission. Thirdly, some information, such as a history of surgery or surgery type, was not input into the final analysis, and this may need to be investigated in future research. Finally, as this study was based on a single center, it will inevitably have sample selection bias, although we did adjust for various confounding factors; for the same reason, we lack external validation of the study. As such, more centers should be enrolled to verify our results.

\section{Conclusions}

In conclusion, this study presents a nomogram, compiled through the use of LASSO regression and logistic regression, which considers various clinical risk factors in evaluating the probability of AKI occurrence in the patients with acute brain injury in the NICU. This scale can help clinical physicians prevent the occurrence of AKI and improve the prognosis of acute brain injury patients.

\section{Acknowledgments}

We would like to thank all the staff in the NICU and medical record department for all the support they have given to this 
study. We would also like to thank Steven Wenham for his assistance in editing the language of the manuscript.

Funding: This work was supported by the National Natural Science Foundation of China (No. 81671221, 81671380), the Tianjin Research Program of Application Foundation and Advanced Technology (No. 14ZCZDSY00179), the Clinical Study of Tianjin Medical University (No. 2017 kylc007, Rongcai Jiang), the Natural Science Foundation of Tianjin (No. 17JCZDJC35900), and the Zhao Yi-Cheng Medical Science Foundation.

\section{Footnote}

Conflicts of interest: The authors have no conflicts of interest to declare.

Etbical Statement: The authors are accountable for all aspects of the work in ensuring that questions related to the accuracy or integrity of any part of the work are appropriately investigated and resolved. The study was approved by The Ethical Committee of Tianjin Medical University General Hospital (No. IRB2019-WZ-123). The authors are accountable for all aspects of the work in ensuring that questions related to the accuracy or integrity of any part of the work are appropriately investigated and resolved.

Open Access Statement: This is an Open Access article distributed in accordance with the Creative Commons Attribution-NonCommercial-NoDerivs 4.0 International License (CC BY-NC-ND 4.0), which permits the noncommercial replication and distribution of the article with the strict proviso that no changes or edits are made and the original work is properly cited (including links to both the formal publication through the relevant DOI and the license). See: https://creativecommons.org/licenses/by-nc-nd/4.0/.

\section{References}

1. Gruber A, Reinprecht A, Illievich UM, et al. Extracerebral organ dysfunction and neurologic outcome after aneurysmal subarachnoid hemorrhage. Crit Care Med 1999;27:505-14.

2. Koeze J, Keus F, Dieperink W, et al. Incidence, timing and outcome of AKI in critically ill patients varies with the definition used and the addition of urine output criteria. BMC Nephrol 2017;18:70.

3. James MT, Hemmelgarn BR, Wiebe N, et al. Glomerular filtration rate, proteinuria, and the incidence and consequences of acute kidney injury: a cohort study. Lancet 2010;376:2096-103.

4. Han SS, Kim S, Ahn SY, et al. Duration of acute kidney injury and mortality in critically ill patients: a retrospective observational study. BMC Nephrol 2013;14:133.

5. Büttner S, Stadler A, Mayer C, et al. Incidence, Risk Factors, and Outcome of Acute Kidney Injury in Neurocritical Care. J Intensive Care Med 2020;35:338-46.

6. Coca SG, Singanamala S, Parikh CR. Chronic kidney disease after acute kidney injury: a systematic review and meta-analysis. Kidney Int 2012;81:442-8.

7. Teasdale G, Jennett B. Assessment of coma and impaired consciousness. A practical scale. Lancet 1974;2:81-4.

8. Khwaja A. KDIGO clinical practice guidelines for acute kidney injury. Nephron Clin Pract 2012;120:c179-84.

9. Wagner M, Wanner C, Schich M, et al. Patient's and physician's awareness of kidney disease in coronary heart disease patients - a cross-sectional analysis of the German subset of the EUROASPIRE IV survey. BMC Nephrol 2017;18:321.

10. Perera GA. Hypertensive vascular disease; description and natural history. J Chronic Dis 1955;1:33-42.

11. Luther MK, Timbrook TT, Caffrey AR, et al. Vancomycin Plus Piperacillin-Tazobactam and Acute Kidney Injury in Adults: A Systematic Review and MetaAnalysis. Crit Care Med 2018;46:12-20.

12. Giuliano CA, Patel CR, Kale-Pradhan PB. Is the Combination of Piperacillin-Tazobactam and Vancomycin Associated with Development of Acute Kidney Injury? A Meta-analysis. Pharmacotherapy 2016;36:1217-28.

13. Zhang Y, Jiang L, Wang B, et al. Epidemiological characteristics of and risk factors for patients with postoperative acute kidney injury: a multicenter prospective study in 30 Chinese intensive care units. Int Urol Nephrol 2018;50:1319-28.

14. Dreischulte T, Morales DR, Bell S, et al. Combined use of nonsteroidal anti-inflammatory drugs with diuretics and/or renin-angiotensin system inhibitors in the community increases the risk of acute kidney injury. Kidney Int 2015;88:396-403.

15. Ejaz AA, Mohandas R. Are diuretics harmful in the management of acute kidney injury? Curr Opin Nephrol Hypertens 2014;23:155-60.

16. Redfors B, Bragadottir G, Sellgren J, et al. Effects of norepinephrine on renal perfusion, filtration and oxygenation in vasodilatory shock and acute kidney 
injury. Intensive Care Med 2011;37:60-7.

17. Connelly J, Benani DJ, Newman M, et al. Systemic effects of low-dose dopamine during administration of

Cite this article as: An S, Luo H, Wang J, Gong Z, Tian Y, Liu X, Ma J, Jiang R. An acute kidney injury prediction nomogram based on neurosurgical intensive care unit profiles. Ann Transl Med 2020;8(5):194. doi: 10.21037/atm.2020.01.60 cytarabine. J Oncol Pharm Pract 2017;23:436-42.

18. Marik PE. Low-dose dopamine: a systematic review. Intensive Care Med 2002;28:877-83. 